\title{
EQUATOR-Oncology: reducing the latitude of cancer trial design and reporting
}

\author{
Habeeb Majeed ${ }^{1}$ and Eitan Amir ${ }^{*}, 1,2$ \\ ${ }^{1}$ Division of Medical Oncology and Hematology, Princess Margaret Cancer Centre, Toronto, ON M5G 2M9, Canada and \\ ${ }^{2}$ Department of Medicine, University of Toronto, Toronto, ON M5S 1A1, Canada
}

Well-designed and appropriately reported clinical trials are essential to evaluate treatment efficacy and form the basis for regulatory approval of new cancer treatments, post-marketing funding decisions, and endorsement by the wider oncology community. In contrast, poorly designed or inadequately reported studies can impair the clinical relevance of these results. Inaccurate or unreproducible data can ill-advise on further study of new treatments, or may result in the inability to translate benefit observed in clinical trials into an improvement in patient outcome in routine practice. Problems which may influence the interpretation of trials include: the use of narrow eligibility criteria that limits generalisability, the use of surrogate end points that have not been validated as reflecting patient benefit, the reporting of statistically significant but clinically less meaningful results, the underestimation of toxicity, and biased reporting - both in the primary publication and by the media (Tannock et al, 2016). To ensure the highest fidelity in clinical research, it is important to have a framework for optimal design and reporting of clinical trials. Although a number of reporting criteria (Schulz et al, 2010; von Elm et al, 2007) have been developed and endorsed by journal editors and the research community, few have focused exclusively on oncology trials.

In this issue, MacCarthy et al, (2018) highlight some of the important issues related to the conduct and reporting of oncology trials and have taken steps to address these issues through a new project, EQUATOR-Oncology. The EQUATOR (Enhancing the QUAlity and Transparency Of health Research) network, established in 2006, is a global initiative that has brought together researchers and journal editors with the aim of achieving accurate, complete, and transparent reporting of health research studies to support research reproducibility and usefulness (EQUATOR Network, 2017a). While the network highlights commonly utilised generic criteria such as the CONSORT (Schulz et al, 2010) and STROBE (von Elm et al, 2007) statements, for the reporting of randomised clinical trials and observational studies, respectively, they also identify issues that continue to limit oncology research reporting (Peron et al, 2013; Maillet et al, 2016; Sivendran, Galsky 2016), necessitating the need for oncology-specific resources. This led the investigators and their collaborators to establish the EQUATOR-Oncology sub-network, which aims to aggregate published literature on oncology research methodology and reporting. While initially the focus of EQUATOR-Oncology has been on reporting standards for oncology clinical trials based on core domains common to all types of clinical trials, the next phase will include guidance for efficient research planning and design. Ensuring robustness in the planning of any research project is a key step in obtaining reliable research findings. The EQUATOROncology network, however, will need to build consensus for the more controversial elements of oncology research, such as the need for broader eligibility criteria more reflective of patients treated in routine practice (Srikanthan et al, 2016) and the appropriate use of informative censoring in cancer trial analyses, thereby avoiding aberrant estimates of treatment effect resulting from censoring rates that are not distributed evenly between comparison arms of trials (Baselga et al, 2012; Templeton et al, 2015). The EQUATOR network will also be surveying stakeholders to identify barriers and concerns related to adequate research design and reporting; this is an important venture, and should serve to facilitate increased uptake of high standards.

We congratulate the authors on this important and necessary endeavour, which should result in higher-quality study design and reporting for oncology clinical trials. However, it is important to note that the availability of published standards is a first step in improving cancer clinical trials. It will also be necessary to promote endorsement among key stakeholders including researchers, journal editors, professional societies, and the wide spectrum of trial sponsors that include both industry and non-profit agencies. The authors inferred uptake of oncology-specific guidelines from a citation search through Web of Science Core Collection Science Citation Index Expanded (SCI-EXPANDED). They showed that two guidelines (Cheson et al, 2003; Dohner et al, 2010) in acute

*Correspondence: Dr E Amir; E-mail: eitan.amir@uhn.ca

Published online 22 February 2018

C 2018 Cancer Research UK. All rights reserved 0007 - 0920/18 
myeloid leukaemia were cited over 1000 times. However, evaluating uptake using citation indices may not accurately measure conformity with reporting standards, and may lead to an exaggerated assessment of the impact of guidelines. Investigators may cite guidelines as justification for methods without providing evidence of compliance with all aspects of the guideline. The support of oncology societies such as the American Society for Clinical Oncology (ASCO) or the European Society for Medical Oncology (ESMO) would be highly desirable and would increase the visibility of the EQUATOR-Oncology network. Additionally, unequivocal endorsement by journal editors would be of value. The work of the EQUATOR investigators has already been bestowed with an award from the Council of Science Editors for improvement of scientific communication through the pursuit of high standards in reporting (EQUATOR Network, 2017b). The investigators will need to use this momentum to seek additional visibility, thereby allowing their recommendations to become mandatory reporting standards for any research study accepted for publication. Adopting such a standard would be critical in promoting ethical conduct, reporting, and publication of oncology research, and would allow the field to continue to advance to the benefit of all stakeholders.

\section{CONFLICT OF INTEREST}

The authors declare no conflict of interest.

\section{REFERENCES}

Baselga J, Campone M, Piccart M, Burris 3rd HA, Rugo HS, Sahmoud T, Noguchi S, Gnant M, Pritchard KI, Lebrun F, Beck JT, Ito Y, Yardley D, Deleu I, Perez A, Bachelot T, Vittori L, Xu Z, Mukhopadhyay P, Lebwohl D, Hortobagyi GN (2012) Everolimus in postmenopausal hormonereceptor-positive advanced breast cancer. N Engl J Med 366(6): 520-529.

Cheson BD, Bennett JM, Kopecky KJ, Büchner T, Willman CL, Estey EH, Schiffer CA, Doehner H, Tallman MS, Lister TA, Lo-Coco F, Willemze R, Biondi A, Hiddemann W, Larson RA, Löwenberg B, Sanz MA, Head DR, Ohno R, Bloomfield CD, International Working Group for Diagnosis, Standardization of Response Criteria, Treatment Outcomes, and Reporting Standards for Therapeutic Trials in Acute Myeloid Leukemia (2003) Revised recommendations of the International Working Group for Diagnosis, Standardization of Response Criteria, Treatment Outcomes, and Reporting Standards for Therapeutic Trials in Acute Myeloid Leukemia. J Clin Oncol 21(24): 4642-4649.

Dohner H, Bennett JM, Kopecky KJ, Büchner T, Willman CL, Estey EH, Schiffer CA, Doehner H, Tallman MS, Lister TA, Lo-Coco F, Willemze R, Biondi A, Hiddemann W, Larson RA, Löwenberg B, Sanz MA, Head DR, Ohno R, Bloomfield CD (2010) Diagnosis and management of acute myeloid leukemia in adults: recommendations from an international expert panel, on behalf of the European LeukemiaNet. Blood 115(3): 453-474.

EQUATOR Network (2017b) EQUATOR Network honoured by the Council of Science Editors. (Accessed 21 October 2017 ). Available at: http:// www.equator-network.org/2017/05/25/council-of-science-editors-award/.

EQUATOR Network (2017a) EQUATOR Network: what we do and how we are organised. (Accessed 21 October 2017). Available at: https:// www.equator-network.org/about-us/equator-network-what-we-do-andhow-we-are-organised/.

MacCarthy A, Kirtley S, de Beyer JA, Altman DG, Simera I (2018) Reporting guidelines for oncology research: helping to maximise the impact of your research. Br J Cancer 118: 619-628.

Maillet D, Blay JY, You B, Rachdi A, Gan HK, Péron J (2016) The reporting of adverse events in oncology phase III trials: a comparison of the current status versus the expectations of the EORTC members. Ann Oncol 27(1): 192-198.

Peron J, Maillet D, Gan HK, Chen EX, You B (2013) Adherence to CONSORT adverse event reporting guidelines in randomized clinical trials evaluating systemic cancer therapy: a systematic review. J Clin Oncol 31(31): 3957-3963.

Schulz KF, Altman DG, Moher D, CONSORT Group (2010) CONSORT 2010 statement: updated guidelines for reporting parallel group randomized trials. Obstet Gynecol 115(5): 1063-1070.

Sivendran S, Galsky MD (2016) Adverse event reporting in oncology clinical trials - lost in translation? Expert Opin Drug Saf 15(7): 893-896.

Srikanthan A, Vera-Badillo F, Ethier J, Goldstein R, Templeton AJ, Ocana A, Seruga B, Amir E (2016) Evolution in the eligibility criteria of randomized controlled trials for systemic cancer therapies. Cancer Treat Rev 43: 67-73.

Tannock IF, Amir E, Booth CM, Niraula S, Ocana A, Seruga B, Templeton AJ, Vera-Badillo F (2016) Relevance of randomised controlled trials in oncology. Lancet Oncol 17(12): e560-e567.

Templeton AJ, Ace O, Amir E, Vera-Badillo F, Ocana A, Pond GR, Tannock IF (2015) Influence of censoring on conclusions of trials for women with metastatic breast cancer. Eur J Cancer 51(6): 721-724.

von Elm E, Altman DG, Egger M, Pocock SJ, Gøtzsche PC, Vandenbroucke JP; STROBE Initiative (2007) The Strengthening the Reporting of Observational Studies in Epidemiology (STROBE) statement: guidelines for reporting observational studies. Lancet 370(9596): 1453-1457. 